\title{
THD Reduction in Multilevel Inverters Using Real-Time Algorithm
}

\author{
Megha Jess Mathew \\ Assistant Professor, Dept: Of ECE,MVJ College of Engineering, Near ITPB, Channasandra, Bangalore-67 \\ 09060101159
}

\begin{abstract}
Multilevel inverters have been widely applied industries. A family of optimal puisewidth modulation $(P W M)$ methods for multilevel inverters, such as step modulation, can generate output voltage with less harmonic distortion than popular modulation strategies, such as the carrier-based sinusoidal PWM or the space vector $P W M$. However, some drawbacks limit the application of optimal PWM. One of such crucial drawback is that the optimal switching angles could not be calculated in real-time and one has to rely on lookup tables with precalculated angles. We propose a novel real-time algorithm for calculating switching angles that minimizes total harmonic distortion (THD) for step modulation. We give a mathematical proof that the output voltage has the minimum THD. We implemented the algorithm on a digital signal processor and provide experimental results that verify the performance of the proposed algorithm.

Keywords-Multilevel inverter, optimal puisewidth modulation (PWM), power conversion, Total Harmonic Distortion (THD), Digital Signal Processing (DSP).
\end{abstract}

\section{Introduction}

INCREASING attention has been paid to multilevel dc/ac in- verters in recent year [1], [2]. Various modulation methods have been developed for multilevel inverters. A very popular method in industrial applications is the classic carrier-based sinusoidal pulse width modulation (SPWM) that uses the phase-shifting technique to reduce harmonics in the load voltage [1], [3], [4]. In SPWM, the states of power semiconductor switches are determined by the comparison between reference signals and sawtooth signals. The determination of a switching instant can be easily carried out in real-time by an analog circuit, a microprocessor or a digital signal processor (DSP). The space vector PWM [5], [6] is also very popular in industries. The calculation to determine switching instants for the space vector PWM is more complicated than that for the SPWM, but it can still be handled by a microprocessor or a DSP.

Another important modulation method for multilevel in-verters is the optimal PWM, which includes step modulation [7]-[ 10], multilevel selective harmonic elimination [11], and optimal combination modulation [12]. With the same switching frequency, voltage quality generated by the optimal PWM is better than that by the popular SPWM or the space vector PWM. The general procedure for implementing optimal PWM is as follows: based on Fourier series analysis, equation sets whose variables are switching angles are built to meet a specific optimization aim, for example the minimization of total harmonic distortion (THD) of the voltage or the elimination of lower order harmonic components of the voltage. An equation set need to be solved with respect to a certain amplitude value of the fundamental voltage component. Generally, the equation sets are nonlinear and transcendental. Several methods, such as the Newton-Raphson iteration method with multiple variables [8], methods based on the theory of symmetric polynomials and resultants [13], and methods based on genetic algorithms [14], [15], have been proposed to solve nonlinear transcendental equation sets. Calculations based on all the methods above are very time-consuming. Therefore, they cannot be done by a microprocessor or a DSP. in real time. They can only done by a computer offline. Switching angles obtained offline have to be stored in a lookup table in a microprocessor or a DSP. Therefore, one of the dominant drawbacks of the above methods is that they cannot be implemented in real-time due to high overhead of the calculations. Another drawback is that data of switching angles stored in the lookup table increase if the required resolution of the fundamental component of the voltage increases.

Several attempts have been made to develop algorithms by which switching angles can be calculated in real-time by a microprocessor or a DSP. In [16], a simple method was proposed to obtain the switching angles in real-time for step modulation. It makes use of the voltage-second areas of the divided reference voltage according to the output voltage levels of the inverter. The method avoids solving a set of nonlinear transcendental equations, but calculates several trigonometric functions. Thus, the calculation based on this method can be done in real-time. However, this method cannot guarantee that the THD of the voltage is minimized or that the lower order harmonic components of the voltage are eliminated.

The THD of the output voltage of the inverter is a measurement of the harmonic distortion, which is expected to be as small as possible in many applications of multilevel inverters. One popular example is the single- 
phase multilevel inverter for the photovoltaic power supply system [17]-[20], in which the THD of the output voltage of the inverter is regarded as a very important measurement of voltage quality [7].।

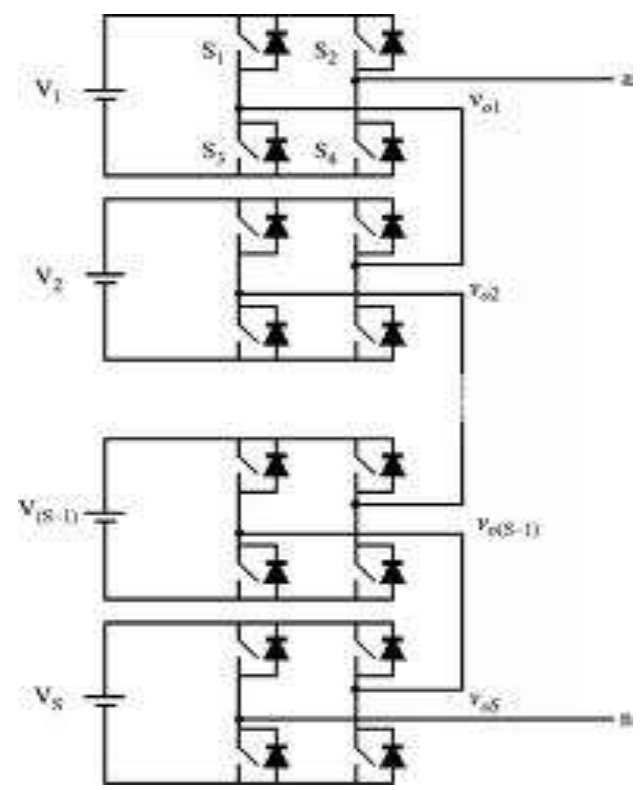

Fig. 1. Cascade multilevel inverter.

This paper proposes a novel method by which the switching angles can be calculated in real-time in the context of step modulation for multilevel inverters. In my project, the optimization aim of the proposed algorithm is the minimization of the Voltage THD. Mathematical derivation is given to prove that the THD is minimized by the proposed algorithm. Comparison with other methods also shows that the THD gained by the proposed method is the smallest. Most importantly, the overhead of calculation is found to be so low that the calculation can be easily done in real-time without any lookup tables. Experimental results are given to verify the performance of the proposed method.

\section{Multilevel Inverters With Step Modulation}

Various topologies, such as diode clamped multilevel inverters and cascade multilevel inverters, have been applied in industries. An important family of modulation methods for multilevel inverters is the optimal PWM. A representative of this family is step modulation. Take the example of the cascade multilevel inverter shown in Fig. 1, where the basic operation principle of the multilevel inverter and the step modulation strategy is explained. As shown in Fig. 1, $v_{\mathrm{Hi}}$ represents the output voltage of the ith $\mathrm{H}$-Bridge (HB). A switching function $F_{i}$, is used to relate $v_{\mathrm{Hi}}$ and $\mathrm{V}_{\mathrm{dc}}$ as shown in

$$
v_{\mathrm{Hi}}=F_{i} \cdot V_{d c}
$$

The value of $F_{i}$ can be either 1 or -1 or 0 . For the value 1 , switches $S H$ and SJ4 need to be turned on. For the value -1 , switches $\mathrm{S}_{i 2}$ and $\mathrm{S}_{i 3}$ need to be turned on. For the value 0 , switches $S_{i 1}$ and $S_{i 3}$ need to be turned on or $S_{i 2}$ and $S_{i 4}$ need to be turned on. The output voltage of the i $\quad \mathrm{n} \quad \mathrm{v} \quad \mathrm{e} \quad \mathrm{r} \quad \mathrm{t} \quad \mathrm{e} r$ $v_{\text {a o is the }}$ summation of the output voltages of HBs. The

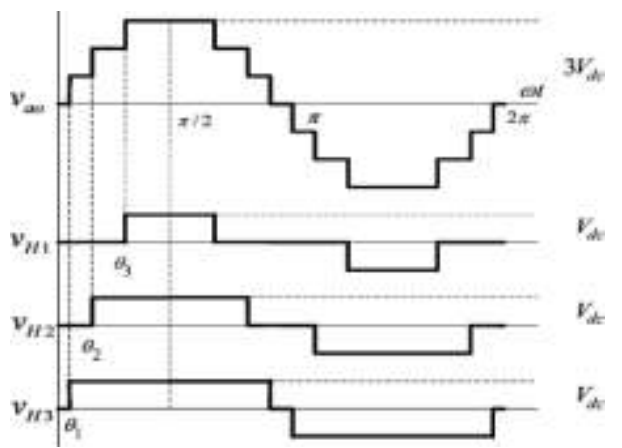

Fig. 2. Output voJlages of the multilevel inverter and its HBs with the step modulation. 
Output voltage of a cascade multilevel inverter with $s$ HBs is expressed as

$$
\mathrm{v}_{\mathrm{ao}}=\sum_{i=1}^{s} \mathrm{v}_{\mathrm{Hi}} \sum_{i=1}^{s} F_{\mathrm{i}} \cdot V_{d c}
$$

Fig. 2 shows the output voltage waveforms of a seven-level cascade multilevel inverter and its HBs under step modulation. $\theta_{1}, \theta_{2}$ and $\theta_{3}$ are switching angles that indicate the on_or off instant of switches in HBs.

By applying Fourier series analysis for a cascade multilevel inverter with $s \mathrm{HBs}$, the amplitude of any odd nth harmonic of $v_{\text {ao }}$ can be expressed as

$$
V n=\frac{4}{n \pi} \sum_{k=1}^{s}\left[V_{d c} \operatorname{Cos}(n \theta k)\right]
$$

Where $n$ is an odd harmonic order and $\theta_{k}$ is the kth switching angle. The amplitudes of all even harmonics are zero. The modulation index $m$ is defined as

$$
m=\frac{\pi}{4} \frac{V_{1}}{V_{\max }}=\frac{\pi}{4} \frac{V_{1}}{s . V_{d c}}
$$

$\mathrm{V}_{\max }$ is the maximum attainable output voltage of the inverter. In the case of the seven-level inverter, $s$ is three and $\operatorname{Vmax}$ is $3 \mathrm{~V}_{\mathrm{dc}}$.

\section{Optimization Aim and Previous Methods to Calculate Switching Angles}

There are different optimization aims for different applications: 1) minimization of the THD of the output voltage of the inverter, for many applications such as photovoltaic systems [18], [19]; 2) elimination of lower order odd harmonics of the load voltage, such as the third, fifth, and seventh ..., for applications equipped with appropriate filters; 3) minimization of the THD but ignoring triple order harmonics that can be cancelled in specific three-phase systems [7]; and 4) elimination of lower order odd harmonics but ignoring the triple order harmonics. This paper focuses on the first aim: minimization of the voltage THD. The amplitude of any odd nth harmonic of the vao is shown in (3). Thus, the voltage THD is expressed as

$$
T H D=\sqrt{\frac{\sum_{n=3,5,7 . \ldots} V_{n}^{2}}{V_{1}^{2}}}
$$

It seems difficult to achieve the minimization of THD directly because the numerator of (5) has infinitely many terms. One solution may be to eliminate only a finite number of harmonics. Generally, one eliminates a few lower order harmonics because they seem to contribute more to THD... In a cascade multilevel inverter with s HBs, only $(\mathrm{s}-1)$ lower order harmonics could be eliminated. Take the example of a seven-level inverter, the third and fifth harmonics can be eliminated as shown in

$$
\begin{gathered}
\cos \left(\theta_{1}\right)+\cos \left(\theta_{2}\right)+\cos \left(\theta_{3}\right)=3 . \mathrm{m} \\
\cos \left(3 \theta_{1}\right)+\cos \left(3 \theta_{2}\right)+\cos \left(3 \theta_{3}\right)=0 \\
\cos \left(5 \theta_{1}\right)+\cos \left(5 \theta_{2}\right)+\cos \left(5 \theta_{3}\right)=0 .
\end{gathered}
$$

Several methods, such as Newton-Raphson iteration method [8], methods based on symmetric polynomials and resultants [13], [15J and methods based genetic algorithms [14], have been proposed to solve the nonlinear transcendental equation set as show in (6). However, calculations based on the above methods are so time consuming that they cannot be done in real time by a microprocessor or a DSP. The switching-angles can only be calculated offline by a computer and the solved angles have to be arranged in the lookup table in a microprocessor or a DSP. Moreover, the above method does not guarantee that the voltage has the minimum THD.

In a cascade multilevel inverter with $\mathrm{s}$ HBs in the context of step modulation, if more than (s - 1) harmonics are considered to minimize THD, these harmonics cannot be eliminated completely since the number of variables is more than the number of equations in the equation set. Minimization of THD in this context has not been investigated until now.

In [16], a simple method was proposed to obtain the switching angles for multilevel inverter in real-time in the context of step modulation. The theory of voltage-second balance is used to determine the switching angles. 
The method avoids solving a set of nonlinear transcendental equations, but calculates several trigonometric functions. Thus, the calculation based on this method can be done in real-time. However, the method, although close, does not provide the true minimum THD.

\section{Proposed Real-Time Algorithm for Calculating Switching Angles}

In this section, we describe a novel algorithm for calculating the switching angles for the multilevel inverter with step modulation. It has two unique characteristics. First, the voltage THD is proven to be minimum (see Section VIII). Second, the calculation is so time efficient that a normal microprocessor or DSP can do the calculation in real-time (see Section V). There are two steps in the algorithm.

Step 1) Determine $\rho$ by solving the equation (for example using Newton-Raphson)

$$
\sum_{k=1}^{s} \sqrt{\left(1-\frac{k-1 / 2}{s-1 / 2} . \rho\right)^{2}}=m . s
$$

Where we recall that $\mathrm{m}$ is the given modulation index and $\mathrm{s}$ is the given number of HBs in the cascade multilevel inverter.

Step 2) Determine the switching angles by evaluating

$$
\theta_{k}=\arcsin \left(\frac{k-1 / 2}{s-1 / 2} . \rho\right), \quad k=1,2, \ldots \ldots ., s .
$$

\section{Analysis of Computational Complexity and Implementation}

Since (7) includes only one variable, $\rho$, it can be quickly solved by the Newton-Raphson method with few iterations. In practice, only one iteration gives a sufficiently accurate value of $\rho$, if the initial value is chosen properly. Computational complexity of the proposed algorithm is analyzed. The Newton-Raphson method is used to solve (7). From (7), we can derive that

$$
\begin{gathered}
f(\rho)=\sum_{k=1}^{s} \sqrt{\left(1-\frac{k-1 / 2}{s-1 / 2} \cdot \rho\right)^{2}}-m . s \\
f^{\prime}(\rho)=\sum_{k=1}^{s} \frac{-\left(\frac{k-1 / 2}{s-1 / 2}\right)^{2} \cdot \rho}{\sqrt{\left(1-\frac{k-1 / 2}{s-1 / 2} \cdot \rho\right)^{2}}}
\end{gathered}
$$

The value of $\rho$ after the $j$ th iteration can be expressed as

$$
\rho_{j}=\rho_{j-1}-f(\rho) / f^{\prime}(\rho)
$$

In most cases, the system is stable and $\mathrm{m}$ changes very slowly. Suppose that $\mathrm{m}$ changes from 0.64 to 0.93 in $58 \mathrm{~ms}$ linearly. The sampling frequency of the DSP program is set as $10 \mathrm{kHz}$. Thus, $\mathrm{m}$ increases around 0.0005 at the next calculation during the next sampling period. The first calculation is for the case of $m$ of 0.64 . At this calculation, the initial value of $\rho$ for the iteration is 0.99 . Four iterations are used to get a precise $\rho$ or the $\mathrm{m}$ of 0.64 , which will be explained later. The next calculation is for the $\mathrm{m}$ of 0.6405 . The initial value of $\rho$ for this iteration is set as solved $\rho$ in the case of $\mathrm{m}$ of 0.64 . Note that only one iteration is used to solve $\rho$ in the case of $\mathrm{m}$ of 0.6405 . Similarly, in the case of $\mathrm{m}$ of 0.641 , the initial value of $\rho$ for the iteration is set as solved $\rho$ in the case of $\mathrm{m}$ of 0.6405 and one iteration is applied to gain $\rho$ in the case of $\mathrm{m}$ of 0.641 . Following this procedure, we calculate all values of $\rho$ for the $\mathrm{m}$ of $0.6405,0.641,0.6415, \ldots$, and 0.93 . In short, at the calculation

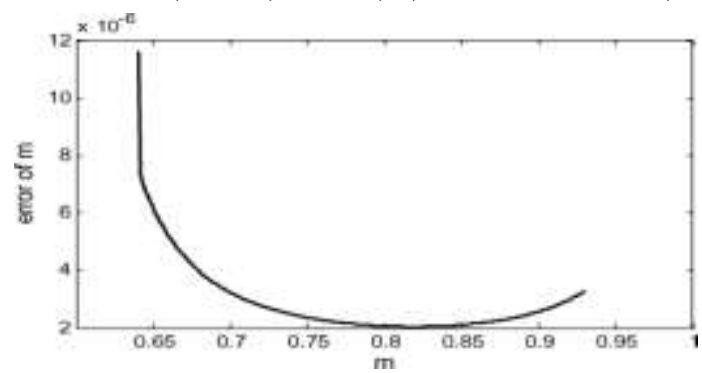

Fig. 3. Error of $m$ after one iteration, where initial value of $p$ is set as the value of $p$ in the last calculation and when $\mathrm{m}$ changes slowly. 


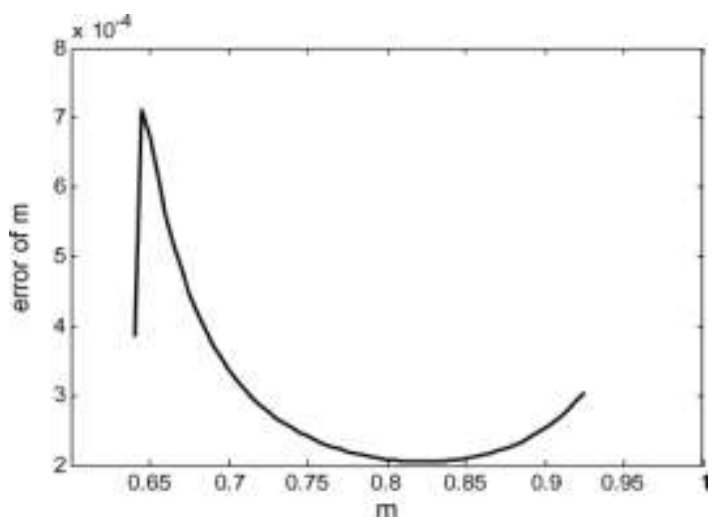

Fig. 4. Error of $m$ after one iteration, where initial value of $p$ is set as the value of $p$ in the last calculation and when $m$ changes quickly.

In a sampling period, the $\rho$ values are calculated by the Newton-Raphson method. Note that the initial value of $\rho$ is set as the calculated $\mathrm{p}$ in the last sampling period. Thus, only one iteration is enough to get the precise value of $\rho$.

The value of $\rho$ derived from the above method is applied in (7) to get a new modulation index, $\mathrm{m}$ ' The difference between $\mathrm{m}$ and $\mathrm{m}$ ' is regarded as the error of $\mathrm{m}$, which indicates the accuracy of the calculation. By the above method, the error of $m$ is very minimal as shown in Fig. 3 .

In dynamic processes, $m$ changes quickly. A satisfactory value of $\rho$ can also be derived by this method in the dynamic processes. Suppose $\mathrm{m}$ changes from 0.64 to 0.93 in $5.8 \mathrm{~ms}$ linearly and the sampling frequency is still set as $10 \mathrm{kHz}$. By this method, the error of $\mathrm{m}$ is less than 0.0008 as shown in Fig. 4.

Computational complexity is analyzed based on the above method by which only one iteration is needed. Only multiplication, division, square root and arcsin operations are considered since they cost most of computing time. The values of $((\mathrm{k}-1 / 2) /(\mathrm{s}-1 / 2))$ and $((\mathrm{k}-1 / 2) /(\mathrm{s}-1 / 2)) 2$ in (9) and (10) are stored in a microprocessor or DSP. The time cost of one iteration is expressed as

$T_{\text {iteration }}=(3+\mathrm{s}) \cdot T_{\text {multiplication }}$

$+T_{\text {rootsquare }}+(\mathrm{s}+1) T_{\text {division }}$

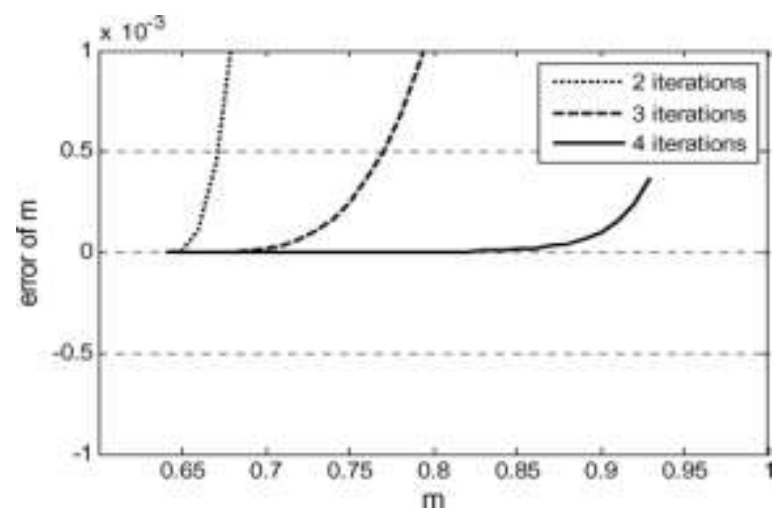

Fig. 5. Error of $m$ after several iterations.

where $T_{\text {multfplication, }}, T_{\text {rootsquarc }}, T_{\text {division }}$ are time costs of multiplication, root square and division operations, respectively. Thus, the total computational complexity of the algorithm is just

$T_{\text {solve }}=(3+2 \mathrm{~s}) \cdot T_{\text {multiplication }}+T_{\text {rootsquare }}$

$$
+(\mathrm{s}+1) T_{\text {division }}+\mathrm{s} . T_{\text {arcsin }}
$$

Where $\mathrm{T}_{\text {arcsin }}$ is time cost of arcsin operation. In the case of a seven-level inverter, $\mathrm{s}$ is three. The computational complexity is just nine multiplications, four divisions, a root square and three arcsin operations. Such computational complexity can be handled by a common microprocessor or DSP easily in real time 
In some special situations, $m$ may have a very quick change, or even a step change. For example, m may have a step change when the operation condition of the system changes. If a very quick change or step change of $m$ is detected, the initial value of $\rho$ for the first iteration is set as 0.99 . The accurate value of $\rho$ can be derived after four iterations. As shown in Fig. 5, the error of $\mathrm{m}$ is less than 0.0005 after four iterations. Thus, in the case of the very quick change or the step change of $\mathrm{m}$, the computational complexity is shown in (14), which can still be handled by a common microprocessor or DSP in real time

$T_{\text {solve }}=(12+8 \mathrm{~s}) \cdot T_{\text {multiplication }}+4 T_{\text {rootsquare }}$

$$
+(4 \mathrm{~s}+4) T_{\text {division }}+\text { s. } T_{\text {arcsin }}
$$

The above analysis is for the implementation in which all calculations are done by the microcontroller or DSP without any lookup tables. The advantage of this implementation is the very high resolution of $m$ and the minimum usage of memory. An alternative implementation is that the values of $\rho$ solved offline by the proposed algorithm are stored in a lookup table, which is suggested by an anonymous reviewer. Thus, switching angles are calculated by (8) and the computational complexity is just s multiplication operations and s arcsin operations.

\section{Comparison of THD With Existing Methods}

In the case of a seven-level multilevel inverter with step modulation, four voltage THDs gained by different methods

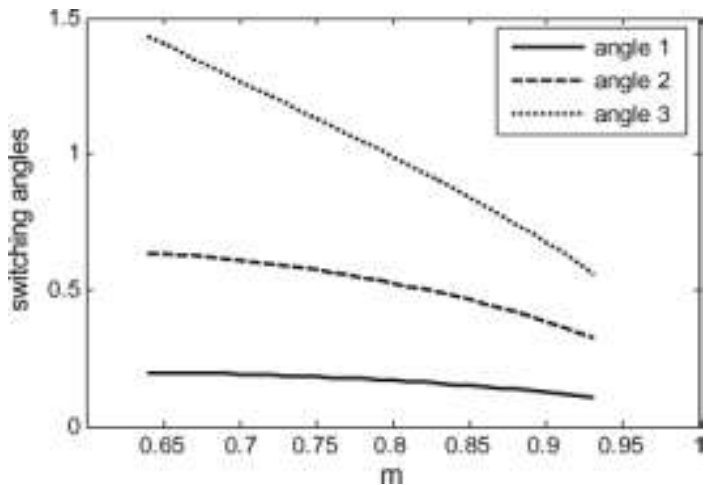

Fig. 6. Switching angles with respect to modulation indexes

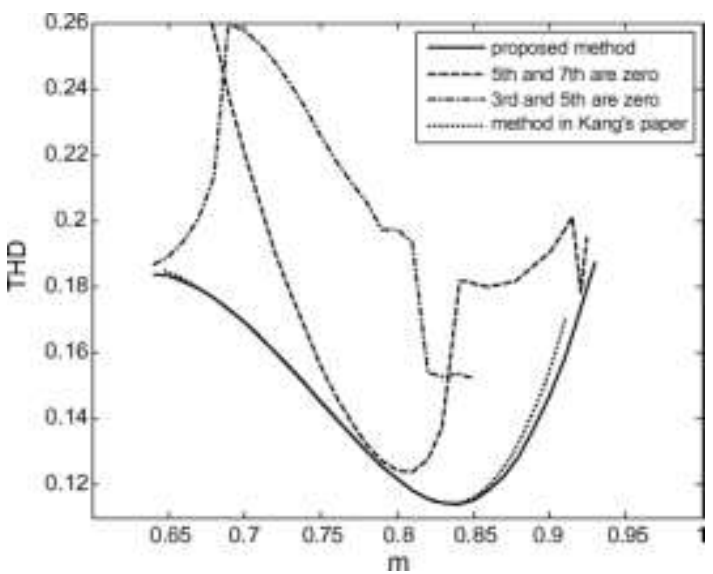

Fig. 7. Comparison of THD between the proposed method and previous methods.

Are compared. The switching angles solved by the proposed algorithm arc shown in Fig. 6. The first THD is gained by the proposed method. The method [8], [13] by which the second THD is gained is to eliminate fifth and seventh order harmonics. The method by which the third THD is gained is to eliminate third and fifth order harmonics. The fourth THD is gained by the method proposed by Kang in [16]. Figs. 7 and 8 show the comparison of results. Large values of the second and third THD suggest that the elimination of lower order harmonics cannot lead to the minimization of THD. The Kang's method based on the theory of voltage second balance [16] can achieve a small THD. However, it is still larger than THD by the proposed method as shown in Figs. 7 and 8 . THD gained by the proposed method is actually proven to be minimal by mathematical derivation in Section VIII. 
Therefore, from the point of view of the voltage THD and real-time implementation, the proposed algorithm is better than previous methods. As shown in Fig. 7, THD is the least when the modulation index is around 0.84, which indicates that the modulation index in normal operations may be designed at 0.84 .

\section{Experimental Results}

To verify performance of the proposed algorithm, a hardware prototype was built in the laboratory as shown in $\mathrm{F}$ $\mathrm{g}$ 9 $\mathrm{T} \quad \mathrm{h}$ $\mathrm{h} \quad \mathrm{r}$

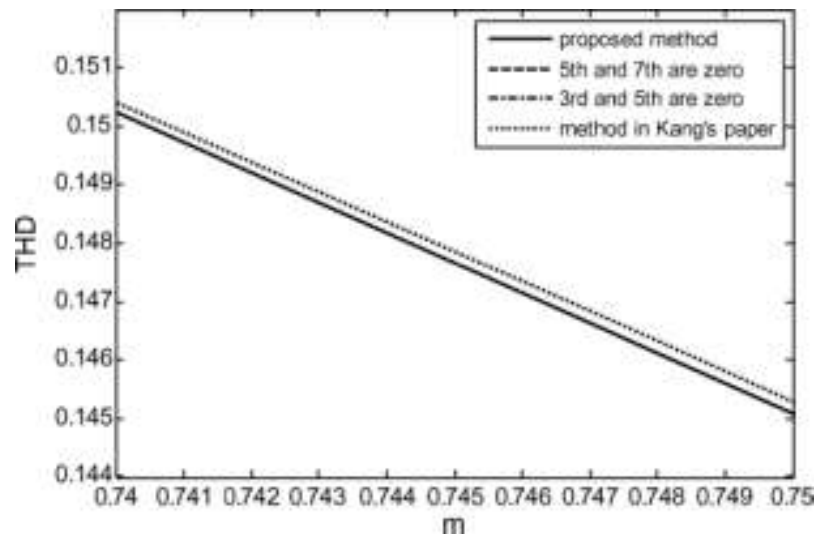

Fig. 8. Detail of comparison of THD between the proposed method and the previous method.

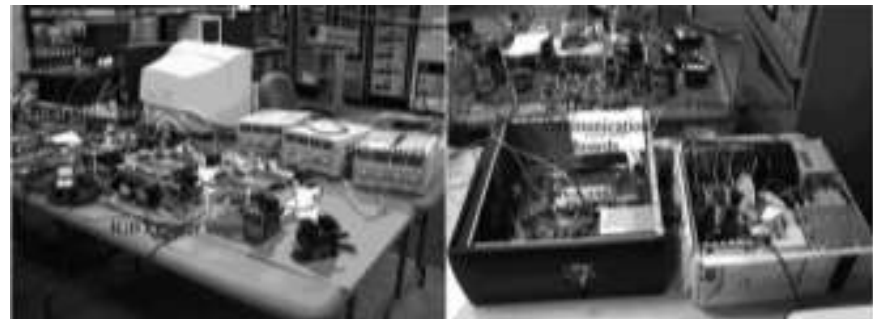

Fig. 9. Experimental prototype.

Insulated-gate bipolar transistor (IGBT) modules are built as three HBs. The dc source of an HB is a dc power supply. A local controller controls an IGBT module through a drive board and optical fibers. The central controller, which is connected to three local controllers, includes a TMS320C6701 DSP board, an AED 106 FPGA daughter board and two PCI boards mounted with an Altera FLEX 10k30A FPGA. In Fig. 9, the left part is the whole picture of the experimental prototype and the right part is the central controller.

The first thing verified by the experiments is that the multilevel inverter can generate a proper output voltage with respect to a stable modulation index. Figs. 10-12 show the output voltages of the inverter and their spectrums with respect to the modulation indexes of 0.7.0.8 and 0.9, respectively. The offline fast Fourier transform analysis of the experimental data shows that the THDs are $17.12 \%, 12.89 \%$ and $14.98 \%$, which are slightly larger than the theoretical values of $16.98 \%, 12.22 \%$, and $14.73 \%$ for the modulation indexes of $0.7,0.8$ and 0.9 , respectively.

The second thing verified by the experiments is that the multilevel inverter can synthesize a proper output voltage with respect to a slowly changing modulation index or a quickly changing modulation indent Figs. 13 and 14 show the output voltages when the modulation indexes change slowly. As shown in Fig. 13, the waveform between two cursors is the output voltage when modulation index changes from 0.64 to 0.93 linearly in $58 \mathrm{~ms}$. Fig. 15 shows the case when modulation index changes from 0.93 to 0.64 linearly in $58 \mathrm{~ms}$. Figs. 15 and 16 show the output voltages while the modulation index changes quickly. As shown in Fig. 15, the waveform between 

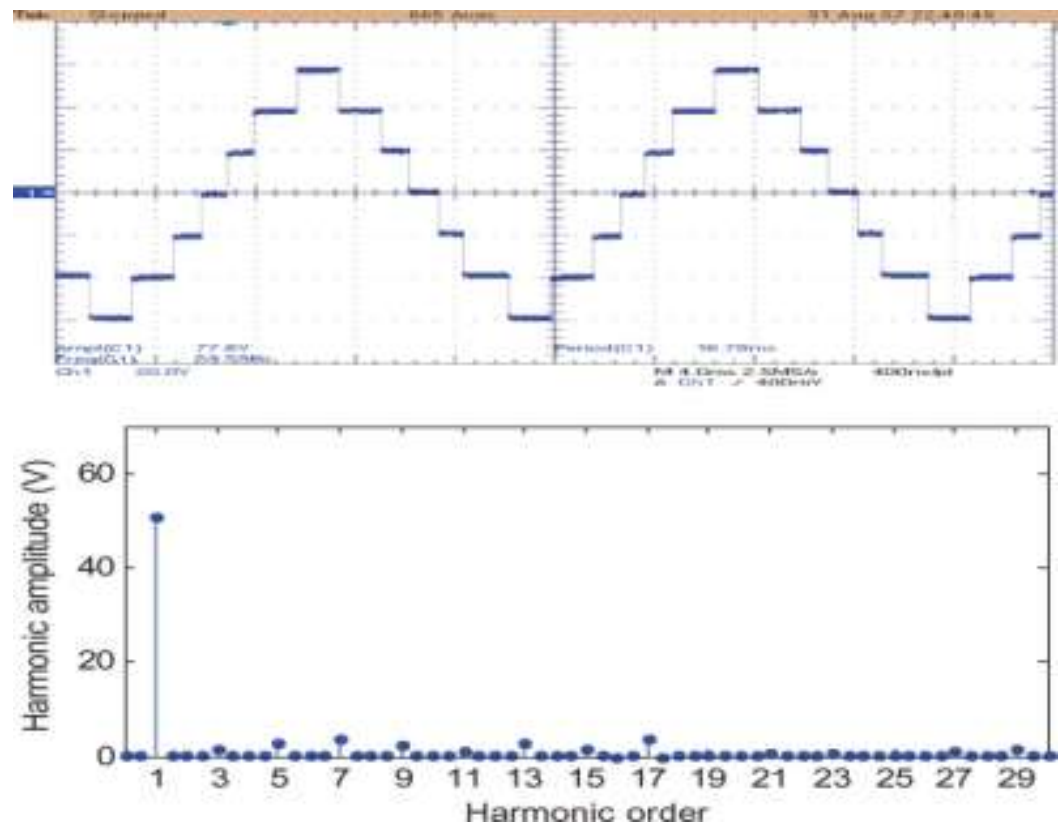

Fig. 10. Output voltage of the inverter and its spectrum when $\mathrm{m}$ is 0.7 .
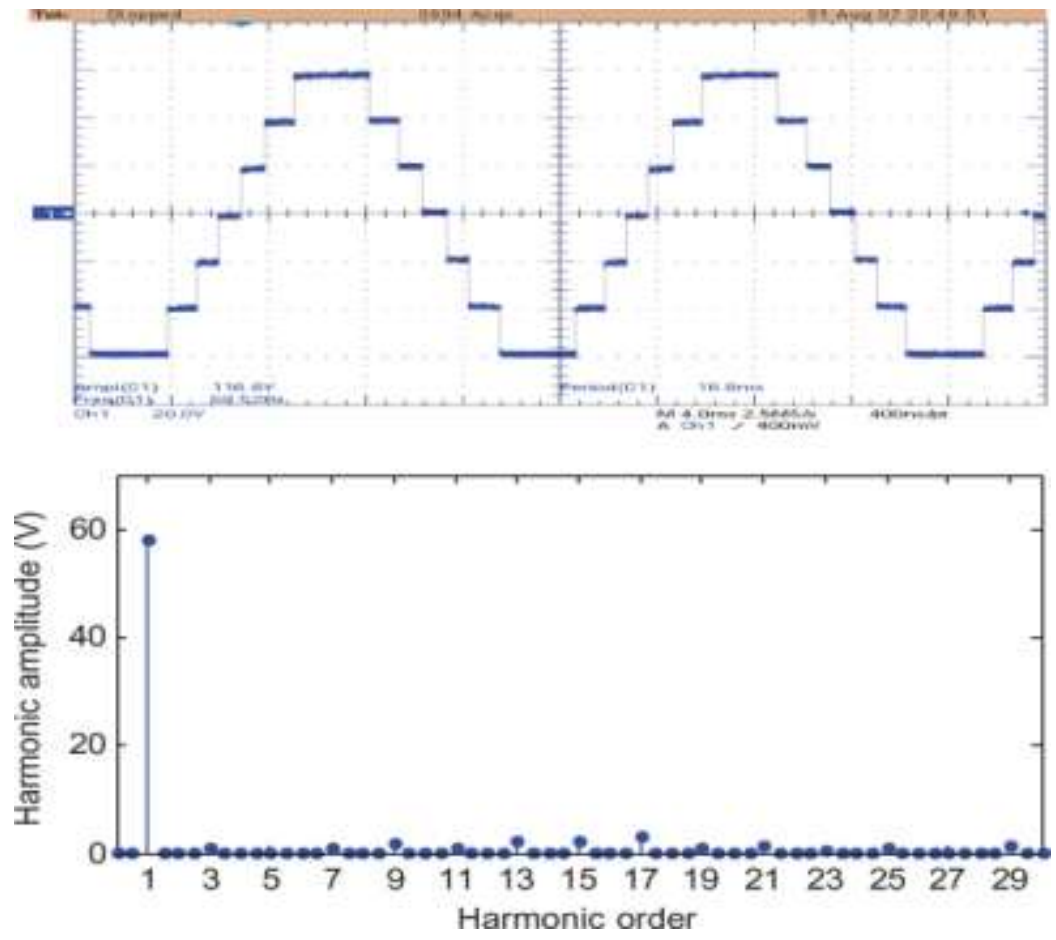

Fig. 11. Output voltage of the inverter and its spectrum when $\mathrm{m}$ is 0.8 .

two cursors is the output voltage when modulation index changes from 0.64 to 0.93 linearly in $5.8 \mathrm{~ms}$. Fig. 16 shows the case when modulation index changes from 0.93 to 0.64 linearly in 5.8 ms. From the results of theoretical calculation as shown in Figs. 3 and 4 and the experimental results as shown in Figs. 13-16, the conclusion can be drawn that the proposed algorithm has high accuracy and that calculation can be done in realtime in the experiments.

The third factor tested by the experiments is how long the calculation can be done by the DSP. The DSP used in the experiment is TMS320C6713, with clock frequency of $225 \mathrm{MHz}$. 

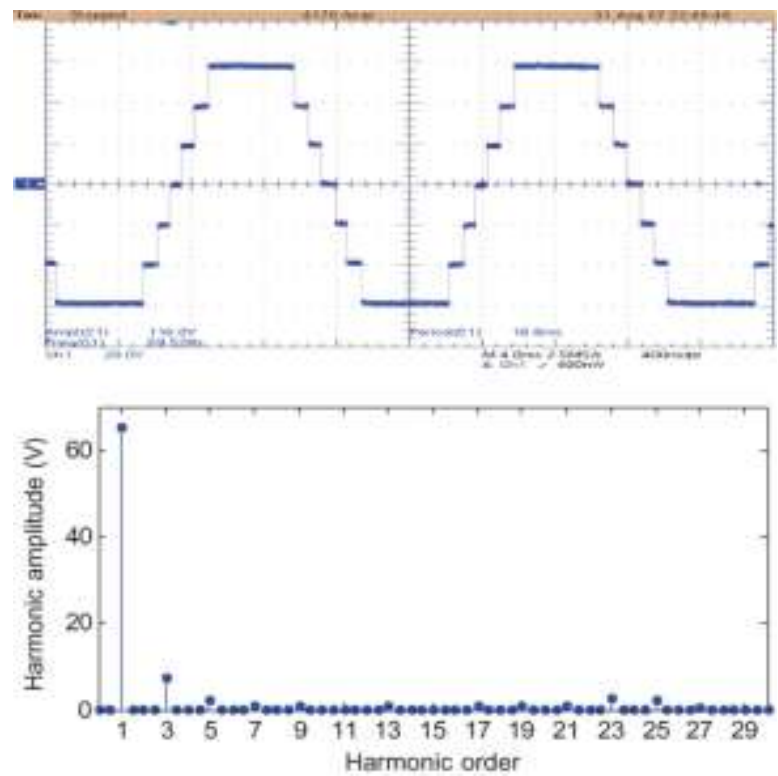

Fig. 12. Output voltage of the inverter and its spectrum when $\mathrm{m}$ is 0.9

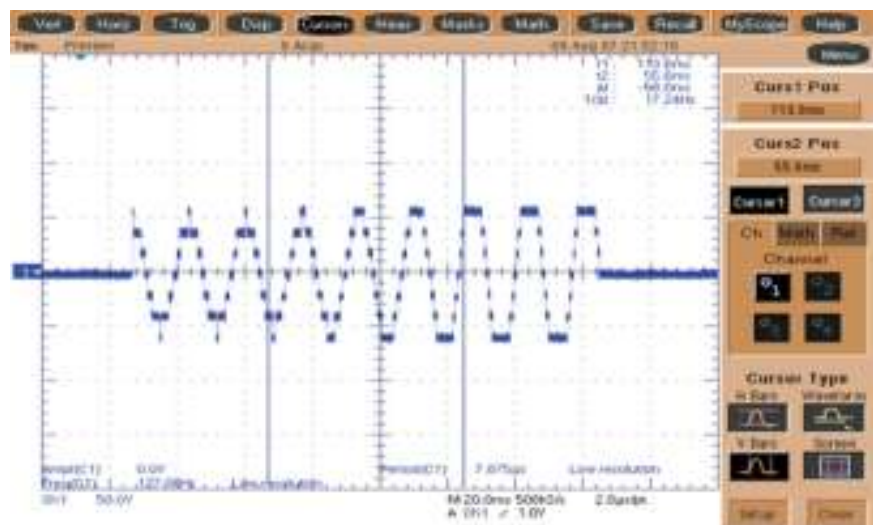

Fig. 13. Output voltage of the inverter when $\mathrm{m}$ changes from 0.64 to 0.93 in $58 \mathrm{~ms}$.

Based on testing results, around 4636 clocks are needed to finish the calculation, i.e., the calculation only costs $20 \mu \mathrm{s}$.

Currently, the functions of asin and square root in the experiment are from libraries provided by DSP software. Within the total 4636 clocks, three times of asin operations cost 2424 clocks and three times square root operations cost 1152 clocks. In the future, calculation time of the proposed algorithm could be decreased significantly by using a specialized algorithm for asin and square root operations.

VIII. Proof of the Proposed Algorithm

The algorithm proposed in Section IV is proven here mathematically. THD is expressed as (5). For simplification,

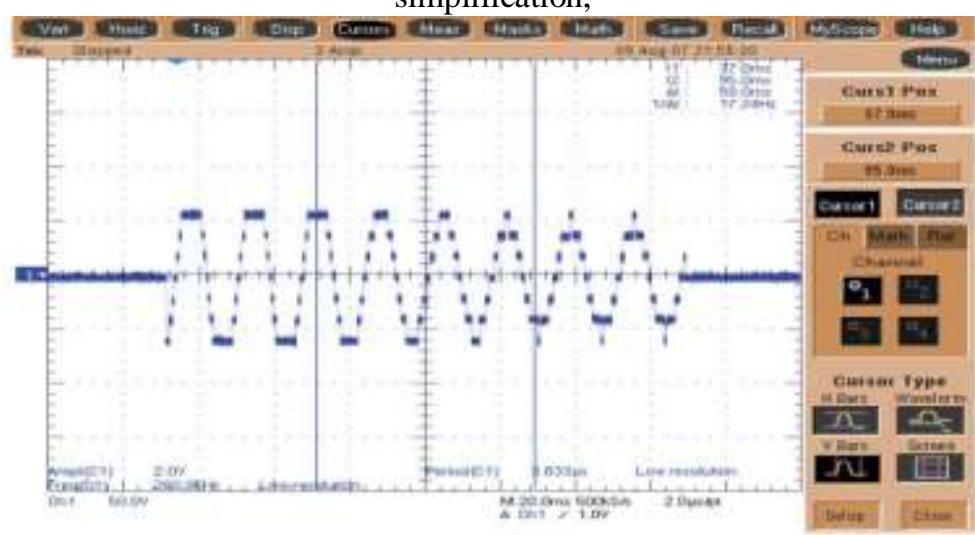

Fig. 14. Output voltage of the inverter when m changes from 0.93 to 064 in $58 \mathrm{~ms}$ 


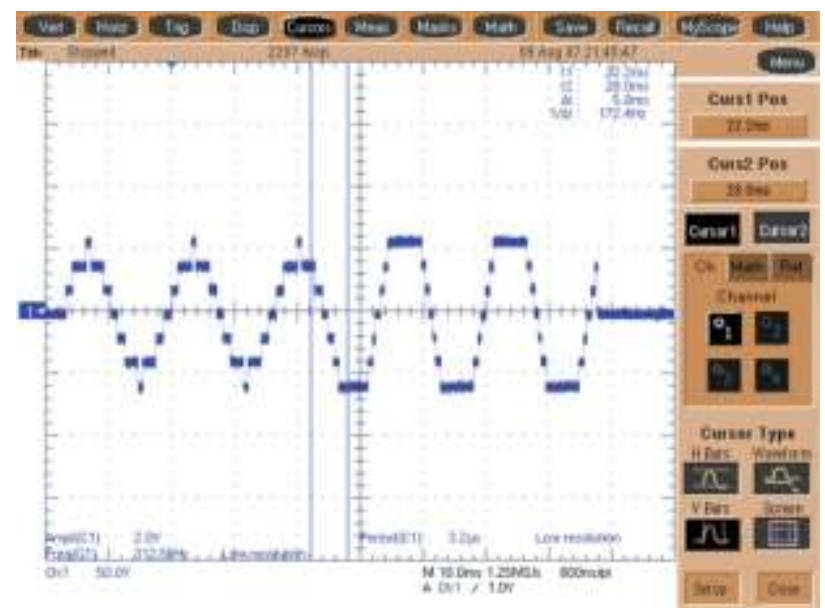

Fig. 15. Output voltage of the inverter when m changes from 0.64 to 093 in $5.8 \mathrm{~ms}$.

THD is expressed as $d$. With the constraint as shown in (4), we will minimize $d^{2}$ instead of $\mathrm{d}$ since it is equivalent and simpler. From the Lagrange multiplier method, we obtain the following system of equations:

$$
\frac{\partial}{\partial \theta_{k}} d^{2}=\lambda \frac{\partial}{\partial \theta_{k}}\left(\frac{\pi}{4} \frac{V_{1}}{s \cdot V_{d c}}-m\right)
$$

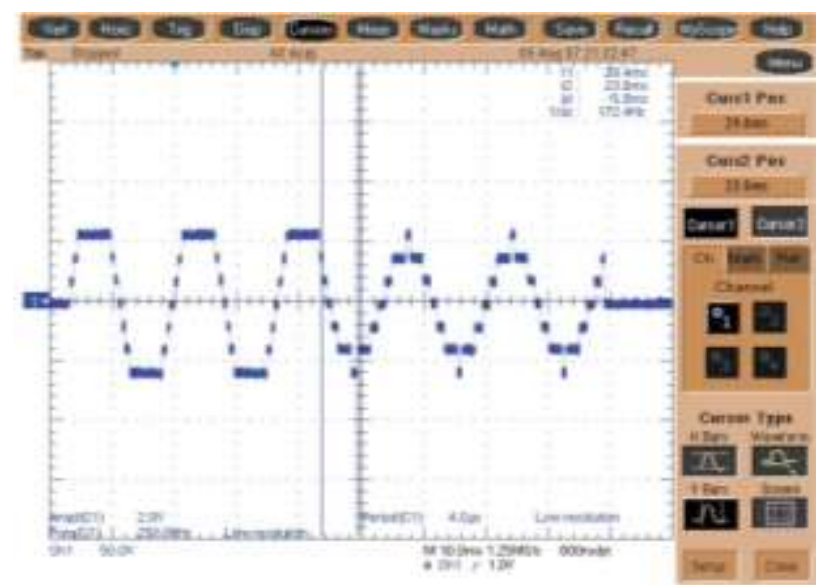

Fig. 16. Output voltage of the inverter when m changes from 0.93 to 0.64 in $5.8 \mathrm{~ms}$

The $n$th harmonic $\mathrm{V}_{n}$ is shown as (3). Note that

$$
\mathrm{RHS}=\lambda \frac{\partial}{\partial \theta_{k}} \frac{\pi}{4 s} \mathrm{~V}_{1}=\lambda \frac{\pi}{4 s V_{\mathrm{dc}}} \frac{4}{\pi}\left(-V d c \sin \theta_{k}\right)=-\frac{\lambda}{\mathrm{s}} \sin \theta_{k}
$$

Note also that LHS can be obtained using (17), which is shown at the bottom of the page. By the Fourier series expansion, we obtain the output voltage of the inverter

$$
v_{\text {ao }}(\theta)=\sum_{\mathrm{n}=1,3,5, \ldots} \mathrm{V}_{\mathrm{n}} \sin \mathrm{n} \theta
$$

By the jump theorem of Fourier series, we have

$$
\begin{aligned}
\sum_{\mathrm{n}=1,3,5, \ldots} \mathrm{V}_{\mathrm{n}} \sin \mathrm{n} \theta_{\mathrm{k}} & =v_{\mathrm{ao}}\left(\theta_{\mathrm{k}}\right) \\
& =\frac{\lim _{\theta \rightarrow \theta_{\mathrm{k}}} v_{\mathrm{ao}}(\theta)+\lim _{\theta \rightarrow \theta_{+\mathrm{k}}} v_{\text {ao }}(\theta)}{2} \\
& =(\mathrm{K}-1 / 2) \mathrm{V}_{\mathrm{dc}}
\end{aligned}
$$


Therefore,

$$
\begin{aligned}
& \text { LHS }=\frac{-\left(\mathrm{k}-\frac{1}{2}\right) V_{\mathrm{dc}}+\sin \theta_{\mathrm{k}} \frac{1}{V_{1}} \sum_{\mathrm{n}=1,3, \ldots . . .} V_{\mathrm{n}}^{2}}{\frac{\pi}{8} V_{1}^{2}} V_{\mathrm{dc}} \\
& \text { LHS }=\frac{\partial}{\partial \theta_{\mathrm{k}}}\left(\frac{\sum_{\mathrm{n}=1,3, \ldots} V_{\mathrm{n}}^{2}}{V_{1}^{2}}-1\right) \\
& =\frac{\left(\frac{\partial}{\partial \theta_{\mathrm{k}}}\left(\sum_{\mathrm{n}=1,3, \ldots . . .} V_{\mathrm{n}}^{2}\right)\right)\left(V_{1}^{2}\right)-\left(\sum_{\mathrm{n}=1,3, \ldots . .} V_{\mathrm{n}}^{2}\right)\left(\frac{\partial}{\partial \theta_{\mathrm{k}}}\left(V_{1}^{2}\right)\right)}{V_{1}^{4}}
\end{aligned}
$$

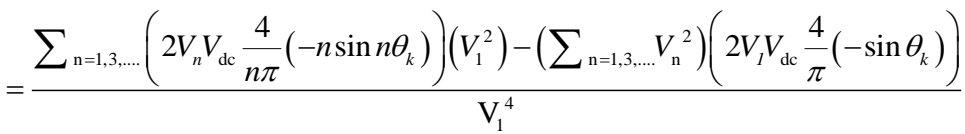

$$
\begin{aligned}
& =\frac{-\sum_{\mathrm{n}=1,3, \ldots .} V_{n} \sin n \theta_{k}+\sin \theta_{k} \frac{1}{\mathrm{~V}_{1}} \sum_{n=1,3, \ldots . . .} V_{n}^{2}}{\frac{\pi}{8} V_{1}^{2}} V \mathrm{dc}
\end{aligned}
$$

By equating LHS and RHS, we have

$$
\begin{aligned}
& \frac{-\left(\mathrm{k}-\frac{1}{2}\right) V_{\mathrm{dc}}+\sin \theta_{\mathrm{k}} \frac{1}{V_{1}} \sum_{\mathrm{n}=1,3, \ldots . .} V_{\mathrm{n}}^{2}}{\frac{\pi}{8} V_{1}^{2}} V_{\mathrm{dc}} \quad=-\frac{\lambda}{\mathrm{s}} \sin \theta_{k} \\
& \frac{\frac{1}{V_{1}} \sum_{\mathrm{n}=1,3, \ldots, \ldots} V_{\mathrm{n}}^{2}+\frac{\lambda}{\mathrm{s}} \frac{\pi}{8} \frac{V_{1} 2}{V_{d c}} \sin \theta_{k}=\mathrm{k}-\frac{1}{2}}{V_{\mathrm{dc}}}
\end{aligned}
$$

Thus,

$$
\begin{gathered}
\sin \theta_{k}=\frac{\mathrm{k}-1 / 2}{\mathrm{~S}-1 / 2} \rho \\
\rho=\frac{(s-1 / 2) V_{\mathrm{dc}}}{V_{1}^{1} \sum_{\mathrm{n}=1,3, \ldots . . .} V_{\mathrm{n}}^{2}+\frac{\lambda}{\mathrm{s}} \frac{\pi}{8} \frac{V_{l}^{2}}{V_{d c}}}
\end{gathered}
$$

In the process above, we pushed the confusing details into p. From (3) and (4), we can get

$$
m . s=\frac{\pi}{4} \frac{V_{1}}{s . V_{\mathrm{dc}}} s=\frac{\pi}{4} \frac{V_{1}}{s \cdot V_{\mathrm{dc}}} \frac{4}{\pi} \sum_{k=1}^{s}\left[V_{\mathrm{dc}} \cos \left(\theta_{\mathrm{k}}\right)\right]=\sum_{k=1}^{s} \cos \left(\theta_{\mathrm{k}}\right)
$$

From (22) and (24), we derive (7), by which the value of $\mathrm{p}$ can be determined.

\section{Conclusion}

It proposed a novel algorithm by which switching angles can be obtained in real-time in the context of step modulation for multilevel inverters. With the algorithm, the voltage THD is minimized. The computational complexity is analyzed to show that the time cost is so negligible that a common microprocessor or a DSP can easily handle it in real-time.

\section{ACKNOWLEDGMENT}

The authors would like to thank the anonymous reviewers for several helpful suggestions such as the analysis of computational complexity in a step change of $\mathrm{m}$ and the alternative implementation of the algorithm. 


\section{References}

[1] J. Rodriguez, J.-S. Lai, and F. Z. Peng, "Multilevel inverters: A survey of topologies, controls, and applications." IEEE Trans. Ind. Electron., vol. 49, no. 4, pp. 724-738, Aug. 2002.

[2] J. M. Carrasco, L. G. Franquelo, J. T. Bialasiewicz, E. Galvan, R. C. PortilloGuisado, M. A. M. Prats, J. i. Leon, and N. MorenoAlfonso, "Power-electronic systems for the grid integration of renewable energy sources: A survey," IEEE Trans. Ind. Electron., vol. 53, no. 4, pp. 1002-1016, Jun. 2006.

[3] X. Xu, Y. Zou, K. Ding, and F. Liu, "Cascade multilevel inverter with phase-shift SPWM and its application in STATCOM," in Proc. IEEE 1ECON, 2004, vol. 2, pp. 1139-1143.

[4] Y. Liang and C. O. Nwankpa, "A new type of STATCOM based on cascading voltage-source inverters with phase-shifted unipolar SPWM,” IEEE Trans. Ind. Appl., vol. 35, no. 5, pp. 1118-1123, Sep/Oct. 1999.

[5] J. Rodriguez, J. Pontt, P. Correa, P. Cortes, and C. Silva, “A new modu-vJ'lation method to reduce common-mode voltages in multilevel inverters,” IEEE Trans. Ind. Electron., vol. 51, no. 4, pp. 834-839, Aug. 2004.

[6] S. Lakshminarayanan, G. Mondal, P. N. Tekwani, K. K. Mohapatra, Sn*^ and K. Gopakumar, "Twelve-sided polygonal voltage space vector based multilevel inverter for an induction motor drive with common-mode voltage elimination," IEEE Trans. Ind. Electron., vol. 54 , no. 5 , pp. $276 \mathrm{i}-2768$, Oct. 2007.

[7] S. Sirisukprasert, J.-S. Lai, and T.-H. Liu, "Optimum harmonic reduction with a wide range of modulation indexes for multilevel converters," IEEE Trans. Ind. Electron., vol. 49, no. 4, pp. 875-881, Aug. 2002.

[8] L. M. Tolbert, F. Z. Peng, and T. G. Habetler, "Multilevel converters for large electric drives," IEEE Trans. Ind. Appl., vol. 35, no. 1, pp. 36-44, Jan./Feb. 1999.

[9] C. Rech and J. R. Pinheiro, "Hybrid multilevel converters: Unified analysis and design considerations," IEEE Trans. Ind. Electron., vol. 54, no. 2, pp. 1092-1104, Apr. 2007.

[10] Y. Liu and F. Luo, 'Trinary hybrid multilevel inverter used in STATCOM with unbalanced voltages, ” Proc. Inst. Electr. Eng.-Elect. Power Appl., vol. 152, no. 5, pp. 1203-1222, Sep. 2005.

[11] L. Li, D. Czarkowski, L. Yaguang, and P. Pi! Jay, "Multileve $\wedge$ selective harmonic elimination PWM technique in series-connected voltage inverters, ” IEEE Trans. Ind. Appl., vol. 36, no. 1, pp. 160-170, JanVFeb. 2000.

[12] Y. Liu, Z. Du, A. Q. Huang, and S. Bhattacharya, “An optimal combination modulation strategy for a seven-level cascade multilevel converter based STATCOM, ” in Conf. Rec. IEEE IAS Annu. Meeting, Oct. 2006, —. pp. 1732-1737.

[13] J. N. Chiasson, L. M. Tolbert, K. J. McKenzie, and Z. Du, "Elimination of harmonics in a multilevel converter using the theory of symmetric polynomials and resultants," IEEE Trans. Control Syst. Technol., vol. 13, no. 2, pp. 216-223, Mar. 2005.

[14] B. Ozpineci, L. M. Tolbert, and J. N. Chiasson, "Harmonic optimization of multilevel converters using genetic algorithms," IEEE Power Electron. Lett., vol. 3, no. 3, pp. 92-95, Sep. 2005. 